\title{
Primera cita de Sympetrum sanguineum (Müller, 1764) (Odonata, Libellulidae) para Gipuzkoa (País Vasco, España).
} First record of Sympetrum sanguineum (Müller, 1764) (Odonata, Libellulidae) in Gipuzkoa (Basque Country, Spain).

Iñaki Mezquita-Aranburu

*

\section{Resumen}

Se aporta la primera cita de Sympetrum sanguineum para la provincia de Gipuzkoa (País Vasco). Palabras clave: Odonata, Sympetrum sanguineum, Península Ibérica, Gipuzkoa.

\section{Abstract}

The first record of Sympetrum sanguineum in Gipuzkoa (Basque Country, Spain) is reported. Key words: Odonata, Sympetrum sanguineum, Iberian Peninsula, Gipuzkoa.

\section{Laburpena}

Gipuzkoako herrialdean (Euskadi, Espainia) Sympetrum sanguineum espeziearen lehen aipamena jasotzen da.

Gako hitzak: Odonata, Sympetrrum sanguineum, Iberiar Penintsula, Gipuzkoa.

\footnotetext{
1 Sociedad de Ciencias Aranzadi. Departamento de Entomología Zorroagagaina 11 • E-20014 Donostia-S. Sebastián, Spain
} 
Sympetrum sanguineum (Müller, 1764) es un libelúlido de tamaño medio que suele habitar en aguas estancadas o de débil corriente con abundante vegetación (Askew, 2004; Dijkstra \& Lewington, 2006). Ocupa un amplio rango de altitud que en el sur de Europa puede llegar a los $1700 \mathrm{~m}$, habitando a esta cota en zonas de turberas alcalinas aunque su presencia es más frecuente y notoria en áreas de baja altitud (Ocharan \& Torralba-Burrial, 2004). Perteneciente al grupo de especies de origen holomediterráneo (Torralba-Burrial \& Ocharan, 2007), está distribuida desde el sudoeste de Europa y el Magreb hasta el oeste de Siberia, faltando en las islas mediterráneas (salvo Sicilia) y Escocia y la mayor parte de Escandinavia (Boudot et al., 2009). Aunque existen poblaciones en todos los territorios limítrofes, tanto en Francia (Pirineos Atlánticos, Grand \& Boudot, 2006) como en Bizkaia (Saloña Bordas \& Ocharan, 1984; Mezquita-Aranburu, 2012), Álava (Ocharan \& Ocharan, 2002; Torralba Burrial \& Mezquita, 2009; Gainzarain et al., 2013) y Navarra (Prunier et al., 2015), su presencia en Gipuzkoa, aunque potencialmente muy probable, aún no se había constatado (MezquitaAranburu, 2009; Mezquita-Aranburu \& Ocharan, 2012). En la península ibérica, la distribución de la especie abarca su mitad septentrional siendo escasa o estando ausente en la mayoría

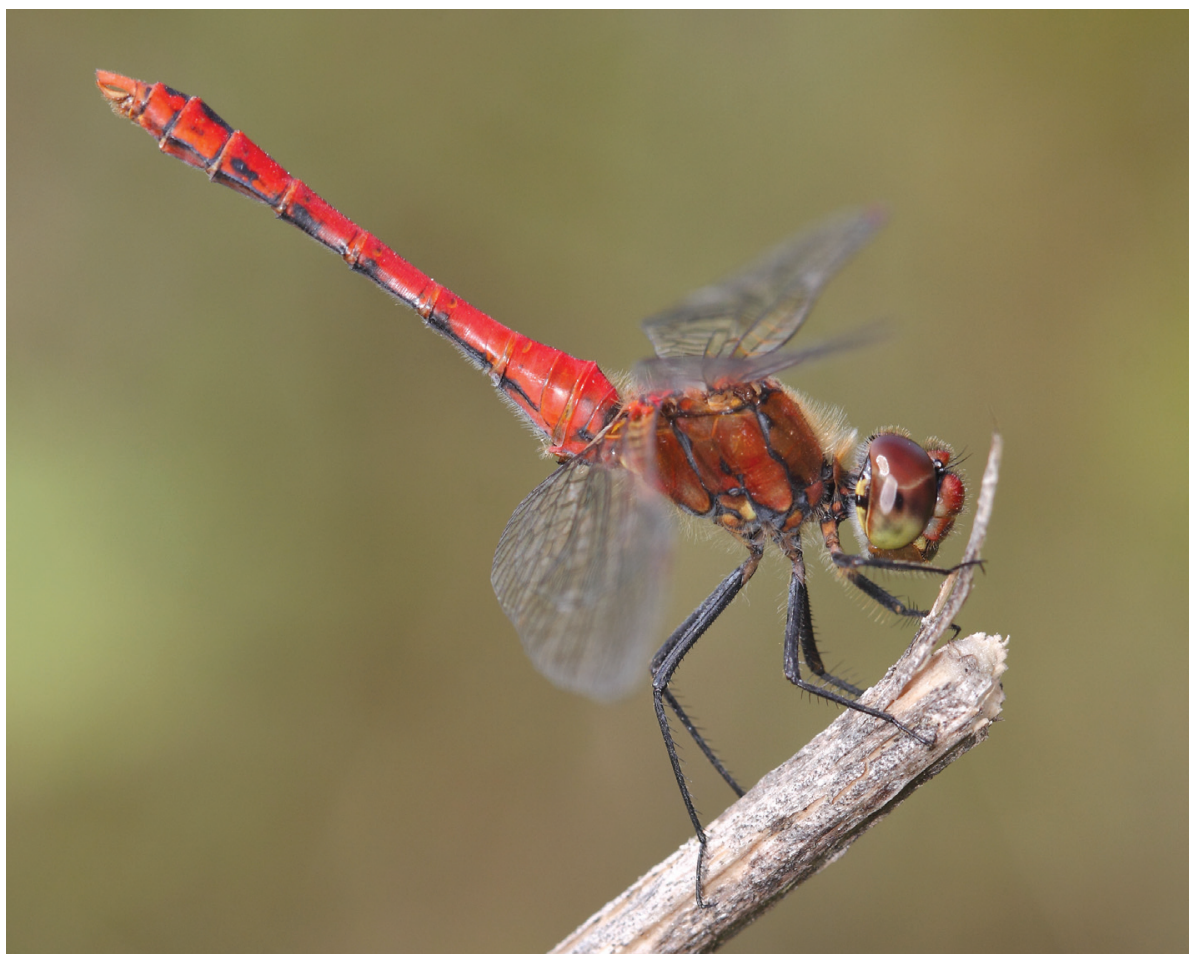

Fig. 1.- Macho de Sympetrum sanguineum localizado en el estanque de Troia (Mutiloa-Gabiria, Gipuzkoa) (foto de IMA).

Fig. 1.- Male of Sympetrum sanguineum observed at Estanque de Troia (Mutiloa-Gabiria, Gipuzkoa) (photo by IMA). 


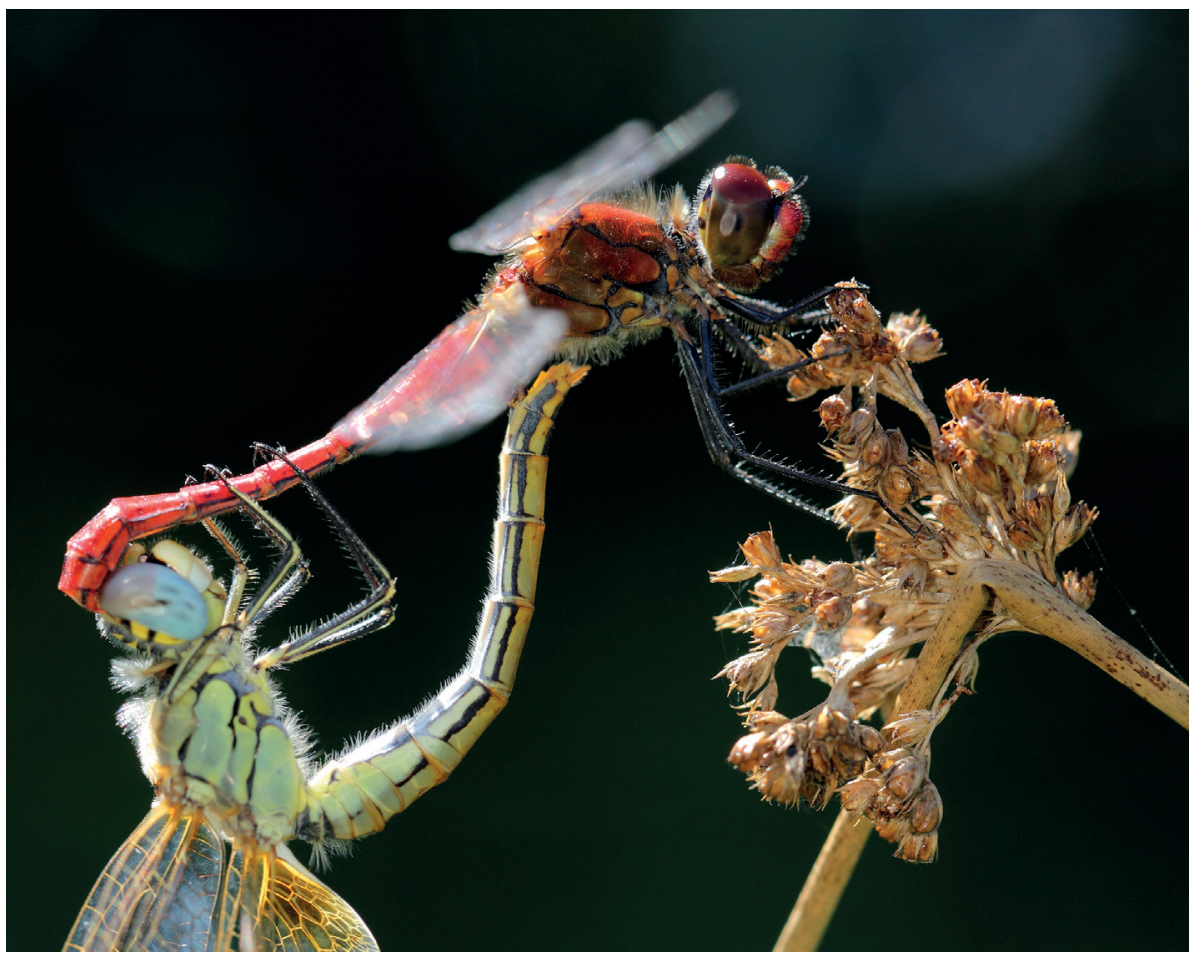

Fig. 2.- Cópula interespecífica de macho de Sympetrum sanguineum y hembra de Sympetrum fonscolombii en el Estanque de Troia (Mutiloa-Gabiria, Gipuzkoa) (foto de IMA)

Fig. 2.- Interspecific mating pair formed by a male Sympetrum sanguineum and a female Sympetrum fonscolombii at Estanque de Troia (Mutiloa-Gabiria, Gipuzkoa) (photo by IMA).

de las provincias de España en su mitad meridional (Prunier et al., 2015), así como en casi todo el centro y sur de Portugal (Maravalhas \& Soares, 2013).

El 28 de agosto de 2012 a las 15:00 (hora oficial) se observó un macho adulto en la Balsa de Troia (275 m s.n.m.), situada entre los términos municipales de Mutiloa y Gabiria (Gipuzkoa), en las proximidades de Ormaiztegi. Se trata de un estanque para el lavado del mineral de una empresa minera actualmente abandonada. El estanque, se nutre de las aguas que aporta la bocamina además de algunos pequeños regueros temporales que vierten en ella sus aguas desde las laderas colindantes. Tras años de inactividad, el paraje ha experimentado un proceso de naturalización destacable que ha hecho posible la presencia de 41 especies de odonatos entre los que cabe destacar Oxygastra curtisii (Dale, 1834) u Onychogomphus forcipatus forcipatus (Linnaeus, 1758), citada recientemente por primera vez para la península ibérica (Mezquita-Aranburu \& Torralba-Burrial, 2015).

La presencia de ganado vacuno y equino con acceso a la ribera ha producido considerables daños en la vegetación riparia a lo largo de estos años siendo el pasado año 2015 el punto 
de inflexión tras el que se tomó la decisión de vallar la zona que da acceso a la ribera sur del estanque. Esta actuación (llevada a cabo por la Diputación de Gipuzkoa) está permitiendo la rápida regeneración de la vegetación ribereña, compuesta principalmente por especies de los géneros Salix, Juncus, Typha, Phragmites y Equisetum.

El ejemplar ocupaba varios posaderos elevados en perchas vegetales muertas, como es habitual en la especie cuando la temperatura del aire es elevada (Askew, 2006), que le permitiría controlar una zona de ribera de unos 40-50 $\mathrm{m}^{2}$. Se tomaron fotografías desde distintos ángulos y posaderos hasta que, sobre las 15:41, se perdió de vista el ejemplar al adentrarse éste en la laguna. En posteriores visitas se confirmó la presencia de la especie en esta misma localización hasta un último avistamiento el día 6 de octubre.

La presencia de la especie ha sido posteriormente comprobada durante los años 2013 (21 de agosto y 30 de septiembre) y 2014 (13 de septiembre), no siendo avistada durante el año 2015. Cabe resaltar que el 21 de agosto de 2013 se pudo fotografiar (Fig. 2) una cópula interespecífica entre un ejemplar macho de S. sanguineum con una hembra de Sympetrum fonscolombii (Selys, 1840). Tras una breve cópula (cuya duración, desde la formación del tándem, rondó en torno a los tres minutos) realizada en una zona sombría y alejada unos diez metros del agua, la pareja se dirigió con vuelo errático hacia la orilla del estanque en dónde la notable falta de coordinación en el vuelo entre ambos individuos dio paso a la separación del tándem.

El año 2016 ha sido observada de nuevo la especie (el 14 de agosto), de nuevo un macho solitario.

Con esta especie, el catálogo de odonatos del Territorio Histórico de Gipuzkoa queda actualizado en 45 especies.

\section{Agradecimientos}

A Antonio Torralba Burrial y José Antonio Gainzarain, por sus valiosas aportaciones que han contribuido a mejorar sensiblemente esta nota. A Juan Carlos Muñoz, por su grata compañía y su gran capacidad para la observación que condujo a la localización de esta especie.

\section{Bibliografía}

- Askew, R.R. 2004. The dragonflies of Europe (revised edition). Harley Books. Colchester.

- Boudot, J.P., Kalkman V.J., Azpilicueta Amorín, M., Bogdanovich, T, Cordero Rivera, A., Degrabiele, G., Domanget, J.L., Ferreira, S., Garrigós, B., Jovic, M., Kotarac, M., Lopau, W., Massrinov, M., Mihokovic, N., Riservato, E., Samraoui, B., Schneider, W. 2009. Atlas of the Odonata of the Mediterranean and North Africa. Libellula, Supplement 9: 1-256.

- Dijkstraa, K-D.B., Lewington, R. 2006. Field guide to the dragonflies of Britain and Europe. British Wildlife Publishing. Dorset. 
- Gainzarain, J.A., Ocharan, F.J., Mezquita, I. 2013. Catálogo de los odonatos (Insecta :Odonata) de Álava, norte de España. Bol. Soc. Entomol. Aragonesa 53 : 173-185.

- Grand, D., Boudot, J.P. 2006. Les libellules de France, Belgique et Luxembourg. Biotope. Mèze.

- Maravalhas, E., Soares, A. 2013. As Libélulas de Portugal. Booky Publisher.

- Mezquita-Aranburu, I. 2009. Gipuzkoako odonatuak / Odonatos de Gipuzkoa. Diputación Foral de Gipuzkoa. Donostia-San Sebastián.

- Mezquita-Aranburu, I. 2012. Libélulas de Bizkaia. BBK. Bilbao

- Mezquita-Aranburu, I., Ocharan, F.J. 2012., Odonatos de Gipuzkoa. Munibe, Cienc. Nat. 60 : 51 75.

- Mezquita-Aranburu, I., Torralba-Burrial, A. 2015. Primera cita de Onychogomphus forcipatus forcipatus (Linnaeus, 1758) (Odonata, Gomphidae) para la Península Ibérica. Bol. SEA 57 : $365-$ 366.

- Mezquita-Aranburu, I., Torralba, A. Ocharan, F.J. 2011. Primera cita de Orthetrum albistylum (Selys, 1848) (Odonata, Libellulidae) para la Península Ibérica. Bol. Asoc. esp. Ent. 35: 519-523.

- Ocharan, R., Ocharan, F.J. 2002. Odonatos del Valle de Cuartango (Álava). Bol. Asoc. esp. Ent. 26: 97-110.

- Ocharan, F.J., Torralba-Burrial, A. 20043. La relación entre los odonatos y la altitud : el caso de Asturias (Norte de España) y la Península Ibérica (odonata). Bol. Soc. Entomol. Aragonesa, 35 : 103-116.

- Prunier, F., Brotóns, M., Cabana, M., Campos, F., Casanueva, P., Chelmick, D., Cordero Rivera, A., Díaz Martínez, C., Evangelio, J.M., Gainzarain, J.A., García-Moreno, J., Lockwood, M., de los Reyes, L., Mañani, J., Mezquita-Aranburu, I., Muddeman, J., Ocharan, F.J., Otero Pérez, F., Prieto Lillo, E., Requena, C., Ripoll, J., Rodríguez Luque, F., Rodríguez, P., Romeo, A., Salcedo, J., Salvador Vilariño, V., Sánchez Balibrea, J., Tamajón Gómez, R, Torralba Burrial, A., Tovar, C., Winter, P., Zaldívar, R. 2015. Actualización del inventario provincial de Odonatos de España peninsular e Islas Baleares. Boletín ROLA 6: 59-84.

- Saloña-Boirdas, M.J., Ocharan, F.J. 1984. Odonatos de Vizcaya. 2. Anisópteros. Cuad. Investig. Biol. 6: 1-10.

- Torralba-Burrial, A., Ocharan, F.J. 2007. Composición biogeográfica de la fauna de libélulas (Odonata) de la Península Ibérica, con especial referencia a la aragonesa. Bol. Soc. Entomol. Aragonesa 41: 179-188.

- Torralba-Burrial, A., Mezquita, I. 2009. Fallos en reconocimiento de pareja en libélulas: cinco tándems intrasexuales inter e intraespecíficos (Odonata: Lestidae, Coenagrionidae y Gomphidae). Bol. Soc. Entomol. Aragonesa 44: 522-524.

- Fecha de recepción/Date of reception: 29-08-2016

- Fecha de aceptación/Date of acceptance: 14-10-2016 